\title{
Tibial Torsion Among Filipinos: A Cadaveric Study
}

\author{
Villamin CAC, MD, Syquia JFC, MD \\ Department of Orthopaedics, University of Santo Tomas Hospital, Manila, Philippines
}

\begin{abstract}
Tibial torsion, the twisting of the tibia about its long axis, can affect rotational positioning in total knee replacement. This angle varies depending on the ethnicity of the subject. There are no published studies to date to determine the tibial torsion among Filipinos. In this study, 28 cadaveric limbs were examined. Our results show that the average tibial torsion among adult Filipinos is $28.9^{\circ}$.
\end{abstract}

Key Words:

Tibial torsion, Filipinos, cadaveric

\section{INTRODUCTION}

Tibial torsion is the twisting of the tibia about its longitudinal axis ${ }^{1}$. In an adult, tibial torsion usually measures $20^{\circ}$ degrees in external rotation ${ }^{2}$. In total knee replacement, rotational positioning of the tibial component is critical since malrotation may lead to complications ${ }^{3}$. Different races have different degrees of tibial torsion due to varying biologic and mechanical factors ${ }^{4}$. There has been no study to date that has looked into tibial torsion among Filipinos. The objective of this study is to determine tibial torsion among Filipinos and to compare our results with those from other studies.

\section{MATERIALS AND METHODS}

Fourteen adult cadavers were obtained from the Department of Anatomy of the University of Santo Tomas, Faculty of Medicine and Surgery. Death certificates were evaluated to verify that the limbs were from Filipinos. For each cadaver, both limbs were dissected and the knee ligaments were cut such that insertions to the tibia were preserved. The interior of the knee was exposed and the following were marked on the tibia: centre of the insertion of the posterior cruciate ligament (PCL); tibial spines; medial border of the patellar ligament; and centres of the insertions of the collateral ligaments. Distally, the malleoli were exposed and a stout pin was passed from the centre of the medial malleolus towards the centre of the lateral malleolus using an anterior cruciate ligament (ACL). targeting guide (Figure 1).

Digital photographs were taken of the specimens with the camera positioned superior to the tibial plateau and with the digital sensor plane perpendicular to the long axis of the bone. The images were downloaded to a personal computer and the following lines were superimposed on the images: a line connecting the centre of the posterior cruciate ligament to the middle margin of the patellar ligament; and a line perpendicular to the intermalleolar pin. The angle formed by the two lines was considered to be the tibial torsion angle (Figure 2).

Descriptive statistics to determine the mean, standard deviation, and range were used for analysis of results. The differences in the results obtained between male and female subjects, and between the right and the left sides, were evaluated using the Student t-test with $\mathrm{p}$ value $<0.05$ as significant.

\section{RESULTS}

There were five females and nine males in the present study. There were a total of twenty-eight limbs dissected. The mean tibial torsion angle was $28.9^{\circ}+/-10.8^{\circ}$ with a range from 10 to $50^{\circ}$. There were no tibiae that were in internal rotation. There was not a statistically significant difference between the left or the right limbs, nor was there any statistical difference between the male or female limbs (Table I and Table II).

\section{DISCUSSION}

Tibial torsion, which is the twisting of the tibia about its longitudinal axis, varies during development and early childhood. It results in a change in alignment of the planes of motion of the knee and the ankle ${ }^{1}$. The adult level of approximately $20^{\circ}$ of external rotation is reached by about five years of age ${ }^{2}$.

Computed tomography imaging has often been used to determine tibial torsion. However, the most accurate technique for measuring tibial torsion is anthropometric measurement of necropsy specimens ${ }^{5}$. This study was therefore designed to use anatomical specimens instead of relying on clinical or radiographic studies.

Even so, there is still no consensus on which proximal and distal landmarks to use when determining the proximal and distal anteroposterior axes of the tibia. Proximally, the tibial tubercle was the landmark that varied more than any other 
Table I: Mean rotational angles in Filipino cadaveric tibia comparing left and right knees

\begin{tabular}{|lccc|}
\hline Tibial Anteroposterior Axis & Right knee & $\begin{array}{c}\text { Rotational Angle } \boldsymbol{\omega} \text { (in degrees) } \\
\text { Left knee }\end{array}$ & p-value \\
\hline 1 & $\begin{array}{c}25.7 \pm 11.8 \\
(\text { range, } 10-50)\end{array}$ & $\begin{array}{c}32.1 \pm 8.9 \\
\text { (range, 13- 43) }\end{array}$ & 0.1217 \\
$\begin{array}{l}\text { *The values are given as the mean and the standard deviation. A positive value of the angle indicates external rotation of the } \\
\text { anteroposterior axis of the distal end of the tibia. }\end{array}$ & \\
\hline
\end{tabular}

Table II: Mean rotational angles in Filipino cadaveric tibia - male and female subjects

\begin{tabular}{|c|c|c|c|}
\hline \multirow[t]{2}{*}{ Tibial Anteroposterior Axis } & \multicolumn{3}{|c|}{ Rotational Angle $\omega$ (in degrees)* } \\
\hline & Male & Female & p-value \\
\hline 1 & $\begin{array}{c}29.1 \pm 10.9 \\
\text { (range, 13-50) }\end{array}$ & $\begin{array}{c}28.6 \pm 11.2 \\
(\text { range, } 10-40)\end{array}$ & 0.9173 \\
\hline
\end{tabular}

Table III: Comparison of tibial torsion in various ethnicities

\begin{tabular}{|lcc|}
\hline Study & \multicolumn{2}{c|}{ Rotational Angle $\boldsymbol{\omega}$, in degrees } \\
& Ave \pm SD (Range) & Ethnicity \\
\hline Turner \& Smillie, 1981 & $19 \pm 4.8$ & Scottish \\
Mizu-uchi et al. 2006 & $16.5 \pm 7.4$ & Japanese \\
& $(1-32)$ & Indian \\
Mullaji et al. 2008 & $21.6 \pm 7.6$ & Filipino \\
Villamin et al. 2011 & $(4.8-39.5)$ & \\
& $28.9 \pm 10.8$ & \\
\end{tabular}

landmark in terms of its location ${ }^{6}$. Other landmarks that have been used for the proximal tibia consist of: a line perpendicular to the posterior tibial margin; a line connecting the middle of the posterior cruciate ligament to the medial border of the patella; a line joining the medial $1 / 3$ of the tibial tuberosity to the centre of a femoral epicondylar line as projected on the proximal tibia; a line connecting the posterior notch of the proximal tibia to either the medial border or the medial one-third of the patellar tendon; a perpendicular line passing through the midpoint of a the clinical epicondylar axis as projected on to the proximal tibia; a line connecting the midpoint of the clinical epicondylar axis as projected on to the proximal tibia to the medial border or medial one-third of the patellar tendon ${ }^{4,7-9}$.

Distally, the anteroposterior axis of the ankle joint has been variously described as a line perpendicular to the anterior cortical margin of the talus, a line perpendicular to a line drawn across the midpoints of the medial and lateral malleoli at the level of the ankle joint, or the axis of the second metatarsal bone $e^{4,7,9}$.

In this study, we used the axis as described by Akagi, et al. to delineate the proximal tibial anteroposterior axis ${ }^{7}$. This is a line connecting the centre of the posterior cruciate ligament to the medial border of the patellar ligament. The femoral trans-epicondylar axis is considered a valid reference for the anteroposterior axes of the femur and the tibia because it approximates the flexion-extension axis of the knee. In the tibia, the axis described by Akagi, et al. is considered to be perpendicular to the femoral trans-epicondylar axis and should therefore represent the anteroposterior axis of the proximal tibia ${ }^{3}$.

We used a line perpendicular to the intermalleolar line as our anteroposterior axis distally. The alignment of the ankle joint is determined by both the tibia and the fibula. Thus, it is more practical to measure tibial torsion by taking both bones into consideration rather than the tibia alone ${ }^{4}$.

Our study shows that the average tibial torsion among Filipinos is $28.9^{\circ}$ of external rotation. This is different from the result obtained in other studies (Table III). Varying values of tibial torsion have been found in different races due to divergent biological and mechanical factors. Racial and geographical variations have been found due to different postures for sitting and varying positional pressures on the leg ${ }^{4}$. An important point to remember when comparing our results with those from other studies is that we used cadaveric specimens while most other studies used either radiographic or clinical measurements. Furthermore, the use of different axes when measuring torsion would naturally result in different measurements.

In the study of Mizuuchi et al. in which 53 osteoarthritic knees in Japanese patients were studied using CT scans, the tibial torsion was $16.5+/-7.4^{\circ}$ (range from $1-32^{\circ}$ ) in external rotation ${ }^{9}$. In this study, the proximal axis was from 


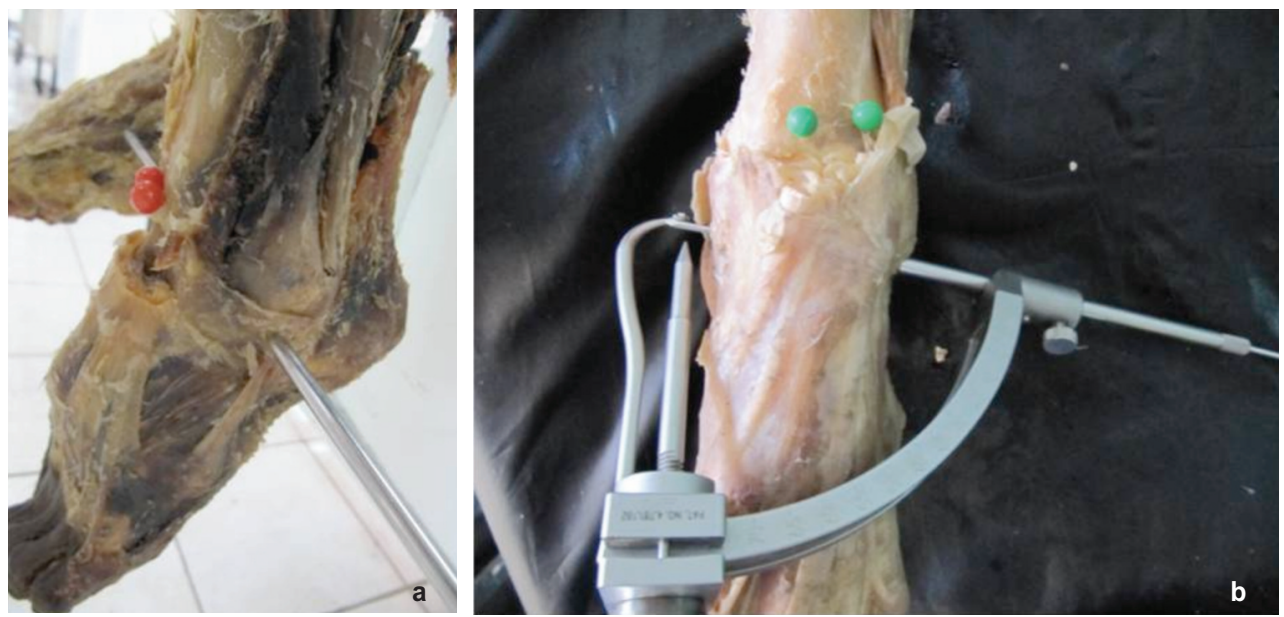

Fig. 1: Cadaveric malleoli were exposed and a stout pin was passed from the center of the medial malleolus towards the center of the lateral malleolus $(A)$ using an anterior cruciate ligament $(A C L)$ targeting guide $(B)$.

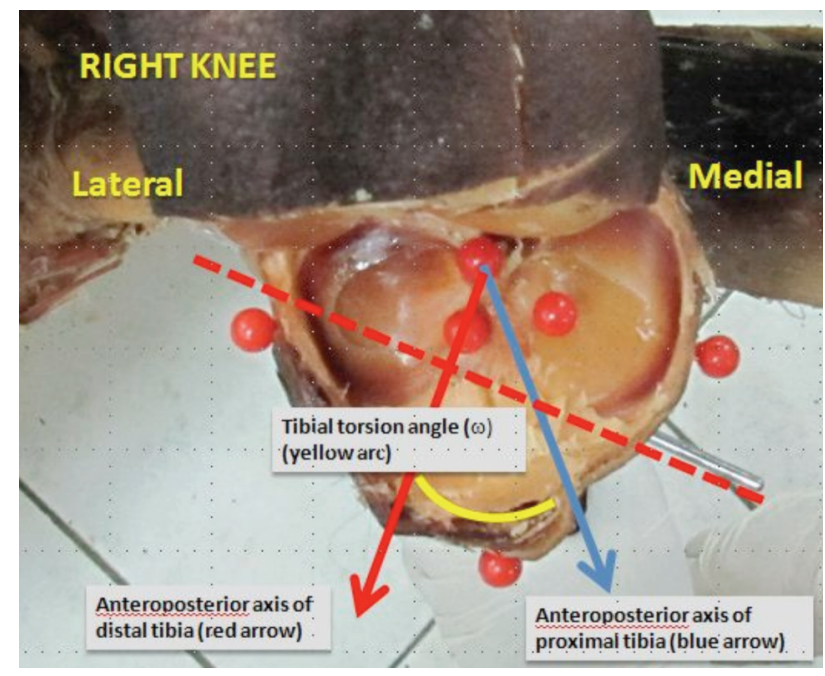

Fig. 2: The angle formed by a line connecting the center of the posterior cruciate ligament to the medial margin of the patellar ligament (blue arrow) and a line perpendicular to the intermalleolar pin (red arrow) was considered to be the tibial torsion angle (yellow arc).

the centre of the PCL to the medial border of the patellar ligament. Distally, the axis was a line perpendicular to the anterior cortical margin of the talus.

In the study by Mullaji et al. in which 100 non-arthritic knees among Indian patients were studied using $\mathrm{CT}$ scans, the average tibial torsion was $21.6+/-7.6^{\circ}$ (range $4.8-39.5^{\circ}$ ) in external rotation ${ }^{4}$. The proximal axis was based on the posterior tibial condylar margin while the distal axis was based on the bimalleolar axis. It has been shown that the posterior tibial margin was internally rotated relative to the trans-epicondylar axis (from which Akagi's line is based) by about $1.6+/-5.1^{10}$. Therefore, the study of Mullaji et al. would give us an external tibial torsional angle of approximately $23.2^{\circ}$ if we were to use the same landmarks used in our study.
In Turner and Smillie's study, a control group of 274 legs from 137 people with no knee complaints was used ${ }^{2}$. The average external tibial torsion was $19+/-4.8^{\circ}$. In this study, a tropometer was used in which the proximal pointer was placed on the tibial tuberosity while the distal pointer was referenced from both malleoli. Although studied in Scotland, no mention is made of the ethnicity of their subjects.

Rotational positioning is critical in total knee replacement because the consequences of malrotation may produce patellofemoral problems, flexion instability, ultrahigh molecular weight polyethylene or post wear, stiffness, and abnormal gait patterns ${ }^{3}$. Excessive internal rotation of the tibial component increases the risk of patellar subluxation and excessive wear of the patella, while excessive external rotation may result in abnormal patellar tracking, aggravation of tibial internal torsion, posterolateral overhang of the tibial component, increased incidence of posteromedial polyethylene wear, and toe-in gait ${ }^{7,11,12}$.

Results obtained from our study could serve as a guide to orthopaedic surgeons when dealing with conditions in which tibial torsion is important, such as when performing total knee replacements and when managing complex deformities and fractures of the tibia.

\section{CONCLUSION}

Tibial torsion among adult Filipinos is externally rotated by about $28.9^{\circ}+/-10.8^{\circ}$ degrees with a range from 10 to $50^{\circ}$. Our results differ than those from other ethnic groups.

\section{ACKNOWLEDGEMENT}

We would like to thank the staff of the Department of Anatomy of the University of Santo Tomas Faculty of Medicine and Surgery for their invaluable assistance in obtaining specimens for this study. 


\section{REFERENCES}

1. Milner C, Soames R. A Comparison of Four In Vivo Methods of Measuring Tibial Torsion. J Anat 1998; 193: 139-44.

2. Turner M, Smillie I. The Effect of Tibial Torsion on the Pathology of the Knee. J Bone Joint Surgery Br, 1981; 63-B (3): 396-8.

3. Aglietti P, Sensi L, Cuomo P, Ciardullo A. Rotational Position of Femoral and Tibial Components in TKA Using the Femoral Transepicondylar Axis. Clin Orthop 2008; 466: 2751-5.

4. Mullaji A, Sharma A, Marawar S, Kohli A. Tibial Torsion in Non-Arthritic Indian Adults: A Computer Tomography Study of 100 Limbs. Indian J Orthop 2008; 42(3): 309-13.

5. Güven M, Akman B, Ünay K, Özturan E, Çakici H, Eren A. (). A New Radiographic Measurement Method for Evaluation of Tibial Torsion: A Pilot Study in Adults. Clin Orthop 2009; 467: 1807-12.

6. Cobb J, Dixon H, Dandachli W, Iranpour F. The Anatomical Tibial Axis: Reliable Rotational Orientation in Knee Replacement. J Bone Joint Surg Br 2008; 90-B(8): 1032-8.

7. Akagi M, Oh M, Nonaka T, Tsujimoto H, Asano T, Hamanishi C. An Anteroposterior Axis of the Tibia for Total Knee Arthroplasty. Clin Orthop 2004; 420: 213-9.

8. Nagamine R, Miyanishi K, Miura H, Urabe K, Matsuda S, Iwamoto Y. Medial Torsion of the Tibia in Japanese Patients with Osteoarthritis of the Knee. Clin Orthop 2003; 408: 218-24.

9. Mizu-uchi H, Matsuda S, Miura H, Higaki H, Ozakazi K, Iwamoto Y. The Effect of Ankle Rotation on Cutting of the Tibia in Total Knee Arthroplasty. J Bone Joint Surg Am 2006; 88-A(12): 2632-6.

10. Bonnin M, Saffarini M, Mercier P, Laurent J, Carrillon Y. Is the Anterior Tibial Tuberosity a Reliable Rotational Landmark for the Tibial Component in Total Knee Arthroplasty? J Arthroplasty 2011; 26(2): 260-7.

11. Eckhoff D, Metzger R, Vandewalle M. Malrotation Associated with Implant Alignment Technique in Total Knee Arthroplasty. Clin Orthop 1995; 321: 28-31.

12. Uehara K, Kadoya Y, Kobayashi A, Ohashi H, Yamano Y. Bone Anatomy and Rotational Alignment in Total Knee Arthroplasty. Clin Orthop 2002; 402: 196-201. 